\title{
Electronic and Optical Properties of the Spiro-MeOTAD Hole Conductor in Its Neutral and Oxidized Forms: A DFT/TDDFT Investigation
}

\author{
Simona Fantacci, ${ }^{*,+}$ Filippo De Angelis, ${ }^{\dagger}$ Mohammad K. Nazeeruddin, ${ }^{\ddagger}$ and Michael Grätzel ${ }^{\ddagger}$ \\ ${ }^{\dagger}$ Istituto CNR di Scienze e Tecnologie Molecolari (CNR-ISTM), c/o Dipartimento di Chimica, Università di Perugia, Via elce di Sotto 8, \\ I-06213 Perugia, Italy \\ ${ }^{\ddagger}$ Laboratory for Photonics and Interfaces, Station 6, Institute of Chemical Sciences and Engineering, School of Basic Sciences, \\ Swiss Federal Institute of Technology, CH-1015 Lausanne, Switzerland
}

Supporting Information

ABSTRACT: The molecular structures and electronic and optical properties of $2,2^{\prime} 7,7^{\prime}$-tetrakis-( $N, N$-di- $p$-methoxyphenyl amine $)-9,9^{\prime}$-spirobifluorene (spiro-MeOTAD) in different oxidation states have been investigated by means of DFT/TDDFT methods. Spiro-MeOTAD has been demonstrated to be an efficient hole-transport material (HTM) in organic lightemitting devices (OLEDs) and in solid-state dye-sensitized solar cells (ssDSCs), and to date spiro-MeOTAD, has yielded the highest ssDSC efficiency. The spiro-MeOTAD radical cation exhibits long-term stability, even though the $2+$ and $4+$ formal oxidation states are accessible. DFT and TDDFT allow the characterization of the excited states involved in the absorption processes of the spiro-MeOTAD-derived cations, an important aspect considering that the oxidized species absorb in the visible region. The excellent agreement between theory and experiment for both neutral spiro-

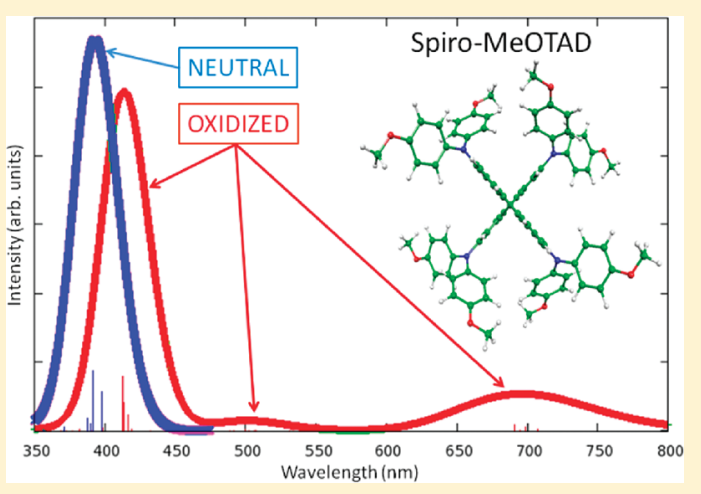
MeOTAD and its oxidized forms opens the possibility for identifying the features that make it an efficient HTM, thus helping in the design of chemically modified or substituted spirobifluorenes.

\section{INTRODUCTION}

The world seems to be undertaking radical changes that will affect all spheres of life. Seeking a clean and sustainable power generation source has given a great boost to the development of new technologies exploiting renewable energies. In this scenario, dye-sensitized solar cells (DSCs) offer a low-cost alternative to directly convert solar light into electricity. Since the inception of DSCs in 1991 by O'Regan and Grätzel, ${ }^{1,2}$ both academic and industrial research efforts have largely focused on improving the efficiency of this type of device, optimizing the different key components and the underlying photochemical processes. ${ }^{2-7}$ The typical DSC architecture consists of two sandwich electrodes of a transparent conductive oxide [fluorine-doped tin oxide (FTO) or indium tin oxide (ITO)]: On the anode is deposited a nanostructured semiconductor metal oxide, which is then sensitized by a molecular dye that serves the function of light absorption and charge separation at the interface between the two materials. ${ }^{4}$ A redox mediator, which can be a liquid or gel electrolyte or a solid hole conductor, plays the important role of regenerating the oxidized dye and transporting the hole toward the cathode, where a catalyst (usually metallic platinum) regenerates the oxidized electrolyte or hole conductor, closing the circuit. ${ }^{8}$ Record DSC efficiencies exceeding $11 \%$ have been obtained using $\mathrm{Ru}$ (II) dyes on $\mathrm{TiO}_{2}$ in combination with a liquid electrolyte based on the $\mathrm{I}^{-} / \mathrm{I}_{3}{ }^{-}$couple in volatile organic solvents. ${ }^{8}$ Despite the high efficiency of the $\mathrm{I}^{-} / \mathrm{I}_{3}{ }^{-}$electrolyte, this redox couple poses several problems in relation to its volatility and corrosive nature toward most metals that impose stringent requirements on cell sealing and module fabrication and eventually might affect the long-term stability and durability of DSC modules for outdoor applications. ${ }^{9}$ Even though a quasisolid gel I ${ }^{-} / \mathrm{I}_{3}{ }^{-}$electrolyte has been shown to considerably enhance the DSC lifetime, partly solving the electrolyte leakage problem and enhancing the cell temporal stability, limits related to metal corrosion by $\mathrm{I}^{-} / \mathrm{I}_{3}{ }^{-}$ remain. In this respect, cobalt(II/III) polypyridyl complexes, ${ }^{10-12}$ ferrocene/ferrocenium $\left(\mathrm{Fc} / \mathrm{Fc}^{+}\right),{ }^{13,14}$ and organic redox couples ${ }^{15}$ are emerging as powerful alternatives to iodide-based liquid electrolytes. Polypyridyl complexes of $\mathrm{Co}$ (II/III) were introduced several years ago ${ }^{10,11}$ and represent interesting candidates as efficient electron-transfer mediators in conjunction with organic dyes. ${ }^{12}$ Also, efficiencies exceeding $7 \%$ have been reported for the $\mathrm{Fc} / \mathrm{Fc}^{+}$redox couple with a novel organic donor-acceptor sensitizer. ${ }^{13}$ Recently, an organic electrolyte based on the disulfide/ thiolate redox couple, namely, the 5-mercapto-1-methyltetrazole ion and its dimer, has been considered promising in rivalling the iodine/triiodide couple. ${ }^{15}$

Received: August 18, 2011

Published: October 18, 2011 
Scheme 1. Spiro-MeOTAD Molecular Structure and Labels of Its Main Geometrical Parameters

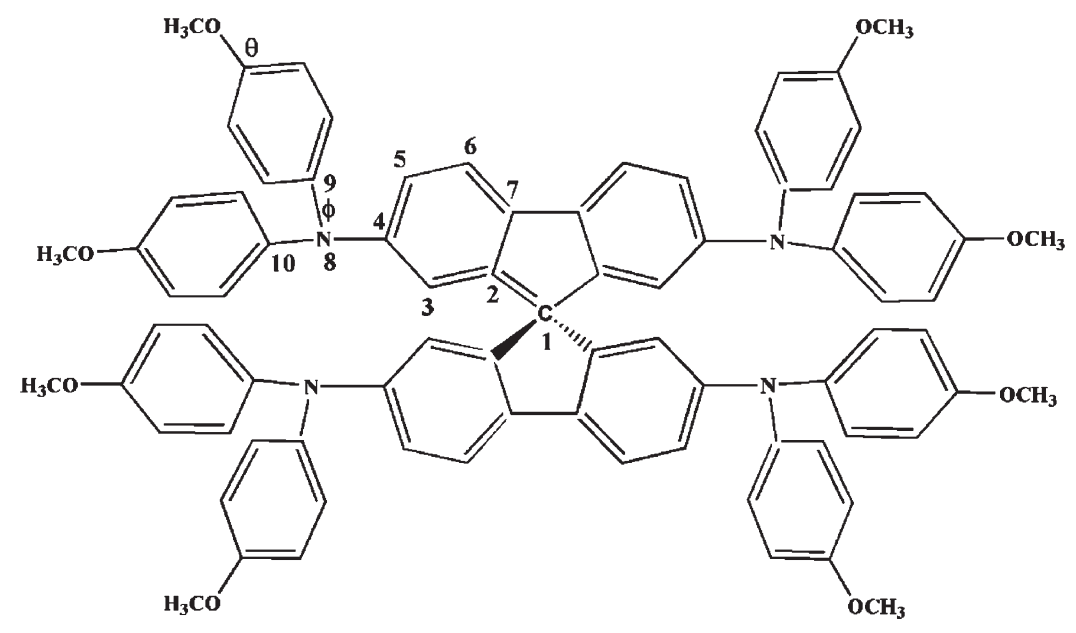

In the past decade, research efforts have focused on replacing liquid DSC electrolytes by solid-state hole-transport materials (HTMs), including inorganic, ${ }^{16,17}$ polymeric, ${ }^{18-23}$ and molecular candidates, $^{24-27}$ as recently reviewed by Yanagida et al. ${ }^{9}$ The most common and efficient HTM used in DSC devices is the $2,2^{\prime} 7,7^{\prime}$-tetrakis-( $N, N$-di- $p$-methoxyphenyl amine)-9, $9^{\prime}$-spirobifluorene system (known as spiro-MeOTAD; see Scheme 1), ${ }^{28,29}$ which has led to top-ranking solid-state DSC (ssDSC) efficiencies. ${ }^{24-27,30-34}$ However, despite considerable progress, ${ }^{25-27,30-38}$ the most efficient ssDSCs still exhibit limited conversion efficiencies of ca. $6 \%^{33}$ compared to devices based on liquid electrolytes. ${ }^{39}$

Record efficiencies for ssDSCs have been obtained using spiro-MeOTAD as the HTM and an indolene-based sensitizer characterized by a high extinction coefficient as the photosensitizer. ${ }^{34} \mathrm{~A}$ major obstacle to reaching higher efficiencies in ssDSCs is mainly the high recombination rate between the oxidized HTM and electrons in the nanocrystalline $\mathrm{TiO}_{2}$, significantly reducing the carrier diffusion length in the cell. ${ }^{40-43}$ To address this problem, thin semiconductor films $(\sim 2 \mu \mathrm{m})$ are necessary, ${ }^{33}$ thus losing out on optical absorption and photocurrent density compared to liquid-electrolyte DSCs, which can employ significantly thicker $\mathrm{TiO}_{2}$ films $(\sim 8-12 \mu \mathrm{m})$. The reason for this nonideal behavior is not yet entirely understood, but incomplete pore filling associated with thicker semiconductor films and lack of screening of the Coulombic attraction between the electrons and holes seem to play important roles. $^{44-46}$

The good performances achieved by ssDSCs using spiroMeOTAD as the HTM result from the combination of its reasonable charge carrier mobility ${ }^{47}$ with its amorphous nature and high solubility, two properties that make it able to infiltrate into mesopourous titania films. Moreover, the presence of the spiro center induces peculiar architectural and electronic properties, enhancing the glass-forming capacity and thus avoiding HTM film crystallization. ${ }^{30}$ The methoxy groups on the peripheral phenyl groups lower the spiro-MeOTAD oxidation potential, thus providing an optimal matching with the dye redox potential. Moreover, the methoxy groups stabilize the oxidized form, preventing the formation of polymeric species, as occurs in triphenylamine (TPA). ${ }^{48}$
Whereas neutral spiro-MeOTAD absorbs light in the UV region of the spectrum $(\sim 390 \mathrm{~nm})$, its oxidized forms present rather strong absorptions throughout the visible range $(\sim 500 \mathrm{~nm})$, extending into the near-infrared (NIR; $\sim 700-900 \mathrm{~nm}) .{ }^{24}$ This is obviously a drawback of the spiro-MeOTAD HTM, as absorption of the oxidized species takes place in the same spectral range where dye sensitizers should absorb light, thus producing an unwanted filtering effect that might reduce the photocurrent output of the cell. In an effort to design new and more transparent HTMs in the oxidized state, a deeper understanding of the optical properties of neutral spiro-MeOTAD and its oxidized forms, identifying the underlying electronic transitions, is essential to design new materials with improved characteristics.

Despite the great experimental interest, to the best of our knowledge, no theoretical investigations have been reported on the optical properties of this HTM, for either the neutral or oxidized state, although a theoretical study has been carried out on the hole-transport properties of spiro-MeOTAD and related compounds. ${ }^{49}$ On the other hand, a density functional theory (DFT)/time-dependent DFT (TDDFT) investigation on pushpull asymmetric spirobifluorenes was recently reported by some of us, focusing on the effects of different acceptor/donor substituents on the linear and nonlinear optical properties. ${ }^{50}$ Here we thus report on the neutral and oxidized species of the spiroMeOTAD, investigating their structural, electronic and optical properties by means of density functional theory (DFT) and time-dependent DFT (TDDFT). The molecular structures of the neutral species and the mono-, di-, and tetra-oxidized species, which have been characterized experimentally, were optimized, and the changes induced by the oxidation processes on the electronic structure and on the absorption spectrum were analyzed. Our results allowed for the assignment of the electronic transitions characterizing neutral and oxidized spiro-MeOTAD, thus unraveling the origins of their visible and near-IR absorption features, eventually allowing for the design of new hole-transporting materials with reduced visible absorption.

\section{COMPUTATIONAL DETAILS}

The molecular structures of spiro-MeOTAD and its oxidized forms (spiro-MeOTAD ${ }^{+}$, spiro-MeOTAD ${ }^{2+}$, and spiro-MeOTAD ${ }^{4+}$ ) 
were optimized in vacuo without any symmetry constraints, using the B3LYP exchange-correlation functional and the 6-31G** basis set for all atoms. All reported calculations were carried out by means of Gaussian 03. ${ }^{51}$ The spin-adapted configurations were considered, and the results for the most stable ones are reported and discussed. Unrestricted DFT calculations, in particular, UB3LYP calculations, were performed for the mono- and dications. Single-point calculations in ethanol were performed including solution effects by a conductor-like polarizable continuum model (C-PCM) as implemented in Gaussian 03. ${ }^{52}$ All calculations were performed without symmetry constraints. The inclusion of solvation effects did not have a relevant impact on the character and energies of the frontier molecular orbitals of spiro-MeOTAD. This conclusion holds also for the characters of the frontier molecular orbitals and the relative energies of the investigated charged species, whereas the absolute energies computed in ethanol were quite different from the values obtained in vacuo because of the different approaches used to describe the positive charge. TDDFT calculations using B3LYP//6-31G** level of theory were performed on all investigated systems. Different numbers of excitations were computed depending on the species investigated, because the oxidized forms show low-lying excited states and, therefore, the description of the main spiro-MeOTAD visible bands requires a large number of states to be calculated, up to 120 excitations for the $4+$ cation. For the investigated systems, we found that inclusion of solvation effects did not sizably affect the calculated excitation energies, providing simulated absorption spectra quite similar to those obtained in vacuo (see Supporting Information); therefore, we report results for TDDFT calculations in vacuo.

\section{RESULTS AND DISCUSSION}

3.1. Geometry, Electronic Structure, and Redox Potentials. The properties that make spiro-MeOTAD an efficient HTM in ssDSCs are its hole-transport capability along with an adequate redox potential for dye regeneration. The investigation of hole conduction is beyond the scope of the present study, and we focus here on the multiple oxidation processes that spiro$\mathrm{MeOTAD}$ might undergo by analyzing the electronic structure of its neutral form and the mono-, di-, and tetracation species and evaluating the oxidation potentials.

In a solution of $0.1 \mathrm{M}$ tetrabutylammoniumtetrafluoroborate in dichlorometane using $\mathrm{Ag} / \mathrm{AgCl}$ in $\mathrm{KCl}(3 \mathrm{M})$ as the reference electrode, two close oxidation peaks were found at 0.65 and $0.75 \mathrm{~V}$, and a third oxidation peak was observed at $\sim 1.0 \mathrm{~V}$. $^{53}$ Cyclic voltammetry measurements in dichlorometane versus the ferrocene/ferrocenium redox couple provided a similar picture: Two one-electron oxidation peaks were observed at 0.12 and $0.23 \mathrm{~V}$, and a two-electron oxidation peak was found at $0.44 \mathrm{~V}^{24}$ A slight change was observed when spiro-MeOTAD was deposited as a film on ITO using $\mathrm{Ag} / \mathrm{AgCl}$ in $\mathrm{KCl}(3 \mathrm{M})$ as the reference electrode, showing the two resolved oxidation peaks to be shifted to slightly less positive values compared to the solution case $(0.55$ versus $0.65 \mathrm{~V}) .{ }^{53}$ The onset of the first oxidation peak of a spiro-MeOTAD film on FTO versus ferrocene/ferrocenium was found at $0.15 \mathrm{~V}$ by Cappel et al., ${ }^{54}$ similar to the solution peak at $0.12 \mathrm{~V}$, even though the two peaks corresponding to the two one-electron oxidations were not observed. ${ }^{54}$ The first oxidation potential of spiro-MeOTAD film deposited into ITO or FTO represents the switch value from an insulating to a conducting state. It therefore becomes crucial to evaluate the oxidation
Table 1. Main Optimized Geometrical Parameters (Mean Values on the Four Quasisymmetric Units) of Spiro-MeOTAD (1) and Its Oxidized Forms $\left(1^{+}, 1^{2+} \text {, and } 1^{4+}\right)^{a, b}$

\begin{tabular}{lcccc}
\multicolumn{1}{c}{ parameter } & $\mathbf{1}$ & $\mathbf{1}^{+}$ & $\mathbf{1}^{2+}$ & $\mathbf{1}^{4+}$ \\
$\mathrm{C}_{1}-\mathrm{C}_{2}$ & 1.533 & 1.533 & 1.534 & 1.539 \\
$\mathrm{C}_{2}-\mathrm{C}_{3}$ & 1.385 & 1.381 & 1.378 & 1.376 \\
$\mathrm{C}_{2}-\mathrm{C}_{7}$ & 1.409 & 1.414 & 1.418 & 1.424 \\
$\mathrm{C}_{3}-\mathrm{C}_{4}$ & 1.409 & 1.414 & 1.421 & 1.428 \\
$\mathrm{C}_{4}-\mathrm{C}_{5}$ & 1.409 & 1.416 & 1.422 & 1.428 \\
$\mathrm{C}_{4}-\mathrm{N}_{8}$ & 1.418 & 1.404 & 1.392 & 1.387 \\
$\mathrm{C}_{5}-\mathrm{C}_{6}$ & 1.393 & 1.387 & 1.382 & 1.377 \\
$\mathrm{C}_{6}-\mathrm{C}_{7}$ & 1.397 & 1.401 & 1.406 & 1.411 \\
$\mathrm{C}_{7}-\mathrm{C}_{7}$, & 1.464 & 1.452 & 1.442 & 1.429 \\
$\mathrm{~N}_{8}-\mathrm{C}_{9}$ & 1.424 & 1.426 & 1.429 & 1.419 \\
$\mathrm{~N}_{8}-\mathrm{C}_{10}$ & 1.422 & 1.425 & 1.428 & 1.424 \\
$\Phi\left(\mathrm{C}_{3} \mathrm{C}_{4} \mathrm{~N}_{8} \mathrm{C}_{9}\right)$ & 142 & 148 & 154 & 154 \\
$\Theta($ angle $)$ & 118 & 119 & 119 & 121
\end{tabular}

${ }^{a}$ Bond distances in angstroms and angles in degrees. ${ }^{b}$ See Scheme 1 for labels.

potential related to the one-electron removal from spiro-MeOTAD. Moreover, because the second potential peak is experimentally located quite close to the first (they differ by only $\sim 0.1 \mathrm{~V}$ ), it is also relevant to investigate the oxidation process of spiro-MeOTAD ${ }^{+}$.

In light of the experimental picture discussed above, we optimized the molecular geometries of spiro-MeOTAD (1) and its experimentally observed oxidized forms $\left(1^{+}, \mathbf{1}^{2+}\right.$, and $\left.\mathbf{1}^{4+}\right)$. The main optimized geometrical parameters are reported in Table 1, and the optimized structure of $\mathbf{1}$ is reported in Figure 1. Neutral spiro-MeOTAD (1) deviates only slightly from $C_{2}$ symmetry, with the four $-\mathrm{N}-(p-\mathrm{OMe}-\mathrm{Ph})_{2}$ ligands almost symmetrically arranged. The spirobifluorene core is planar, whereas the $\mathrm{MeO}-$ substituted phenyl groups assume a propeller-blade-like geometry, with the two $\mathrm{MeO}$ phenyls rotated by ca. $40^{\circ}$ with respect to the reference spiro plane.

The formation of the $\mathbf{1}^{+}$radical cation leads to a doublet ground state. Further removal of one electron leads to the $\mathbf{1}^{2+}$ diradical, for which we calculated the open-shell triplet state to be $6.2 \mathrm{kcal} / \mathrm{mol}$ more stable than the corresponding closedshell singlet. We speculate that the open-shell singlet lies slightly below the triplet, although we focus on the triplet state, which allows for the calculation of the absorption spectra by TDDFT. The tetracation $\mathbf{1}^{4+}$, on the other hand, has a closed-shell singlet ground state.

Comparison of the optimized geometries of spiro-MeOTAD in its neutral and oxidized forms shows rather modest variations in the geometrical structure. This is reflected in the small reorganization energy calculated for the neutral species $\mathbf{1}$ on going to $1^{+}$, which was computed here to be $1.8 \mathrm{kcal} / \mathrm{mol}$. The spirobifluorene core geometry responds to the stepwise oxidations with a decrease of the $\mathrm{C}_{7}-\mathrm{C}_{7}^{\prime}$ bond distance that connects the two phenyl rings of the same fluorene (from $1.464 \AA$ for the neutral species to $1.429 \AA$ for the tetracation) and a slight shortening (lengthening) of the $\mathrm{C}_{1}-\mathrm{C}_{2}\left(\mathrm{C}_{2}-\mathrm{C}_{7}\right)$ bond distance and symmetrically similar variations in the other fluorene moiety. This is in line with the electronic structure discussed below, showing a highest occupied molecular orbital (HOMO) of 1 of antibonding character with respect to the $\mathrm{C}_{7}-\mathrm{C}_{7}{ }^{\prime}$ bond (see Figure 3). Also, the $\mathrm{C}_{4}-\mathrm{N}_{8}$ bond distance, connecting the nitrogen 

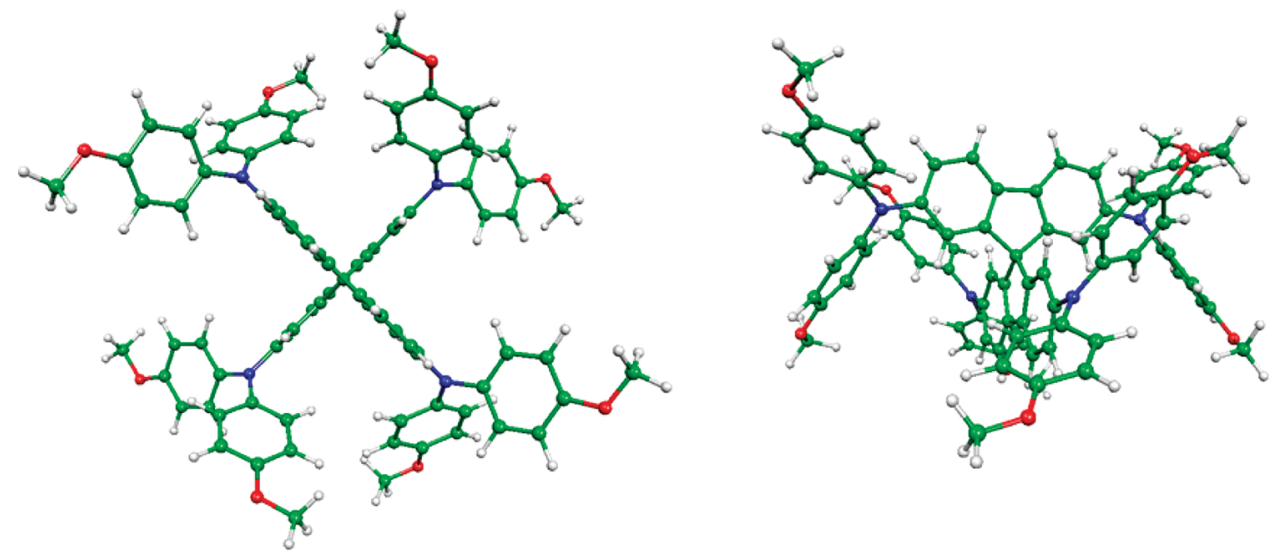

Figure 1. Optimized molecular structure of spiro-MeOTAD in two different views.

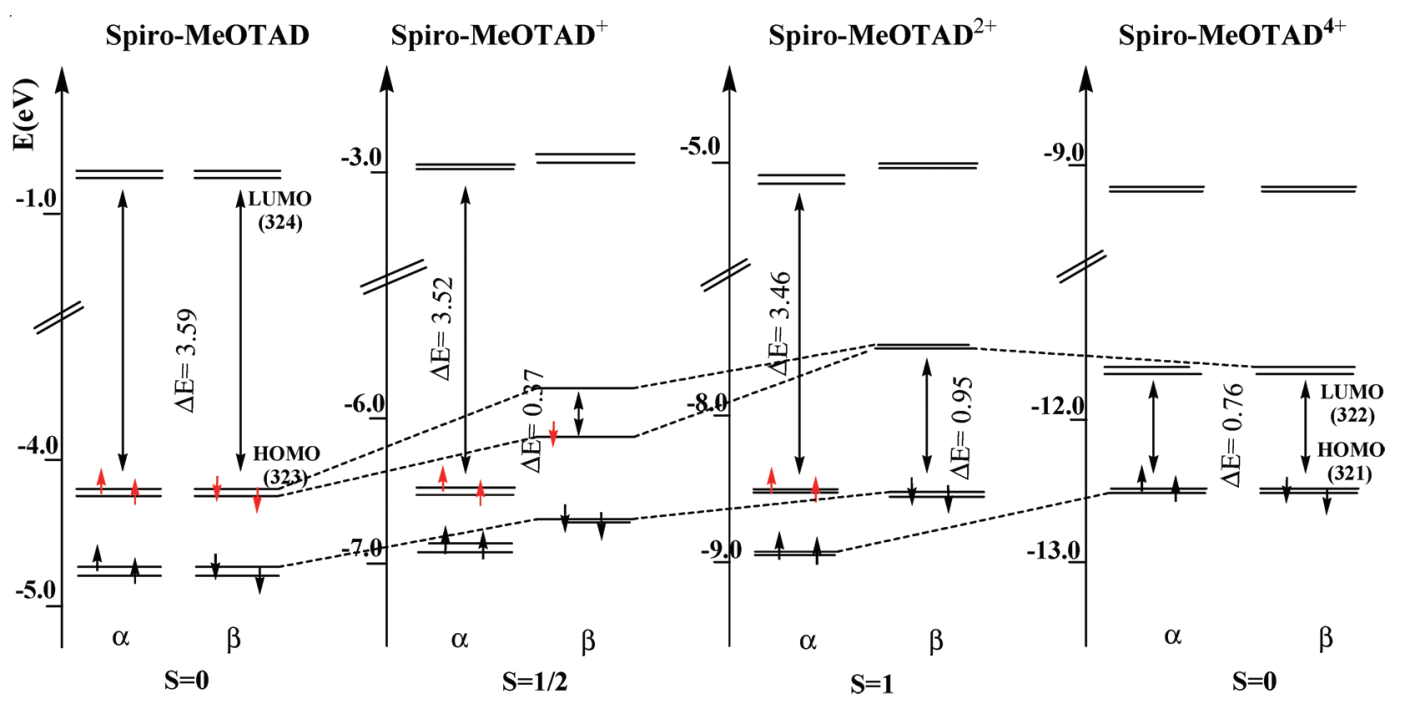

Figure 2. Schematic representation of the molecular frontier orbitals of spiro-MeOTAD, spiro-MeOTAD ${ }^{+},{\text {spiro- }-\mathrm{MeOTAD}^{2+} \text {, and spiro-MeOTAD }}^{4+}$. Red arrows indicate the active electrons. For the sake of clarity, the different energy scales of the four investigated species have been aligned to show the same HOMO energy.

atom to the $\mathrm{MeO}$-substituted phenyls, decreases by $0.03 \AA$ upon four-electron oxidation. The dihedral angle $\Phi$, characterizing the out-of-plane distortion of the $\mathrm{MeO}$-substituted phenyls with respect to the fluorene moieties, was found to increase upon oxidation from $142^{\circ}$ to $154^{\circ}$. The small calculated reorganization energy, in line with the delocalized nature of the oxidation process, is suggestive of a facile hole-transfer process from the oxidized dye to the spiro-MeOTAD hole conductor. In fact, ultrafast hole-transfer kinetics have been observed in ssDSCs employing spiro-MeOTAD. ${ }^{34}$

The electronic structure of spiro-MeOTAD and its changes upon oxidation were thus analyzed. In Figure 2, a schematic representation and correlation of the frontier molecular orbitals of all the investigated spiro-MeOTAD neutral and cationic species is presented, where the red arrows indicate the active electrons, and in Figure 3, the isodensity plots of a selection of molecular orbitals of the neutral species are collected.

The neutral spiro-MeOTAD shows two quasidegenerate highest occupied molecular orbitals ( $\mathrm{HOMO} / \mathrm{HOMO}-1)$, roughly delocalized across the entire molecule, with larger contributions
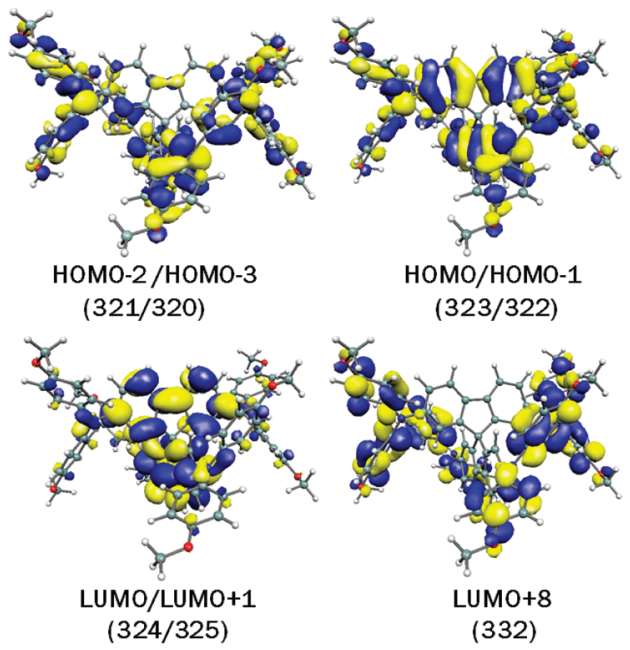

Figure 3. Isodensity plots of the main frontier orbitals of the neutral spiro-MeOTAD species. 
coming from the phenyls of the spirobifluorene core and the nitrogen lone pairs (see Figure 3). On the other hand, HOMO - 2/ $\mathrm{HOMO}-3$, stabilized by ca. $0.5 \mathrm{eV}$ with respect to the $\mathrm{HOMO}$, are mainly delocalized on the outer phenyl rings, with sizable contributions from the nitrogen lone pairs (see Figure 3). HOMO/ HOMO - 1 represent the two redox-active orbitals of spiro$\mathrm{MeOTAD}$ containing four electrons (see Figure 2). Thus, the $1+$ to $4+$ oxidation implies removal of the four electrons contained in $\mathrm{HOMO} / \mathrm{HOMO}-1$. Because of the delocalized nature of these orbitals (see Figure 3), we expect that the stepwise oxidation processes involve the entire molecular framework, with maximum involvement of the phenyl groups of the spirobifluorene moiety. The lowest unoccupied molecular orbitals (LUMO/LUMO + 1) have $\pi^{*}$ character and a spatial distribution over the spiro core. At higher energies, unoccupied orbitals completely delocalized on the entire system are found, namely, LUMO + 6 destabilized by ca. $0.9 \mathrm{eV}$ with respect to the LUMO, or localized on the $-\mathrm{N}(p-\mathrm{OMe}-$ $\mathrm{Ph})_{2}$ ligands, namely, LUMO +8 in Figure 3. The HOMOLUMO gap for this system was calculated as $3.59 \mathrm{eV}$.

Upon the first oxidation process, the radical cation $\mathbf{1}^{+}$is characterized by a doublet ground state. As a result of the unrestricted DFT calculation, orbitals belonging to the $\alpha$ and $\beta$ manifolds have different spatial wave functions and therefore different energies (Figure 2). The HOMO and LUMO of $\mathbf{1}^{+}$belong to the $\beta$ manifold, corresponding to the HOMO -1 and HOMO of the neutral species (see Figures 2 and 3). The HOMO within the $\alpha$ manifold is stabilized by $0.5 \mathrm{eV}$ with respect to the corresponding $\beta$ orbital, still showing its almost degenerate counterpart corresponding to the HOMO -1 of the neutral species. The separation between the HOMO and LUMO of the $\alpha$ manifold is essentially unchanged compared to the neutral species: 3.52 versus $3.59 \mathrm{eV}$.

In the $1^{2+}$ triplet state (see Figure 2), we found two singly occupied molecular orbitals of $\alpha$ spin that are almost degenerate and correspond to the HOMO/HOMO - 1 couple of the neutral spiro-MeOTAD; the LUMO within the $\alpha$ manifold corresponds to the LUMOs of the neutral and singly oxidized species and is located $3.46 \mathrm{eV}$ above the HOMOs. Within the $\beta$ manifold, $\mathrm{HOMO} / \mathrm{HOMO}-1$ show the same spatial wave functions as $\mathrm{HOMO}-2 / \mathrm{HOMO}-3$ of the neutral species and are destabilized compared to these reference orbitals by ca. $0.7 \mathrm{eV}$, whereas $\mathrm{LUMO} / \mathrm{LUMO}+1$ resemble the HOMO/HOMO 1 couple of the neutral species.

The $1^{4+}$ species, which represents the product of the fourelectron oxidation process, has a closed-shell singlet ground state, with the degenerate pair $\mathrm{HOMO}$ and $\mathrm{HOMO}-1$ corresponding to the $\mathrm{HOMO}-2 / \mathrm{HOMO}-3$ couple of the neutral species and the degenerate $\mathrm{LUMO} / \mathrm{LUMO}+1$ couple lying $0.76 \mathrm{eV}$ above the HOMO (see Figure 2) and showing a character similar to that of $\mathrm{HOMO} / \mathrm{HOMO}-1$ of the neutral spiro-MeOTAD (see Figure 3 ). The LUMO + 2/LUMO + 3 couple was found to lie $3.27 \mathrm{eV}$ above the HOMOs and to correspond to LUMO/ LUMO +1 of the neutral species.

To evaluate the oxidation potentials $\left(\Delta G_{\text {ox }}\right)$ associated with the first and second oxidation processes, we added the solvation free energies to the Gibbs free energies computed in vacuo (obtained by a frequency calculation). The results are compared to the negative of $\mathrm{HOMO}$ energy in solution, $-\varepsilon_{\mathrm{HOMO}}$, exploiting Koopmans' theorem in Table 2.

As can be noticed, our calculated values of $\Delta G_{\text {ox }}$ for the neutral and oxidized species show a separation of $0.21 \mathrm{eV}$ between the first two oxidations, in excellent agreement with the separation of the first two one-electron oxidations found experimentally.
Table 2. Calculated Negative HOMO Energy and $\Delta G_{\text {ox }}(\mathrm{eV})$ for Spiro-MeOTAD and Spiro-MeOTAD ${ }^{+}$in Solution

\begin{tabular}{lcc} 
& $-\varepsilon_{\text {Hомо }}$ & $\Delta G_{\text {ox }}$ \\
spiro-MeOTAD & 4.42 & 4.27 \\
spiro-MeOTAD $^{+}$ & 4.84 & 4.48 \\
\hline
\end{tabular}

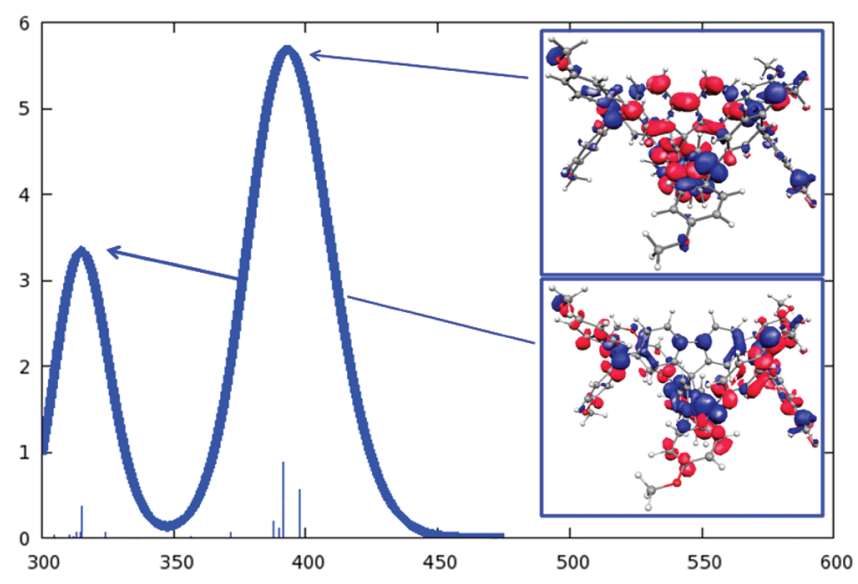

Figure 4. Simulated absorption spectrum of spiro-MeOTAD, showing the energy and related oscillator strength of the electronic transitions. Insets: Isodensity plots of the electron density differences between the excited state and the ground state associated with the most intense transition of the visible absorption band (top) and UV absorption band (bottom). A blue (red) color indicates a decrease (increase) of the electron density upon excitation.

On the technical side, we note that the HOMO values calculated in solution also nicely follow the trend of calculated $\Delta G_{\text {ox }}$ values. A comparison between calculated and experimental values is not straightforward, as the energy of the reference electrode used in cyclic voltammetry experiments with respect to the vacuum level must be known. It seems, however, that our calculated values are slightly underestimated (i.e., less positive) than experimentally available data; considering the electrode at $-4.44 \mathrm{eV}$ versus vacuum would lead to calculated oxidation potentials close to 0 versus normal hydrogen electrode (NHE) to be compared to experimental values more positive than NHE by ca. $0.5 \mathrm{~V} .^{53,54}$

3.2. Absorption Spectra of Spiro-MeOTAD and Its Oxidized Forms. Spiro-MeOTAD works in ssDSCs as an HTM; nevertheless, great interest is focused on the optical properties of its neutral and oxidized forms, with the aim of comprehending the nature of the main transitions responsible for the absorption spectra. In particular, as mentioned before, the oxidized spiro-MeOTAD forms absorb in the visible region, thus competing with the dye for light absorption. The absorption spectrum of the neutral spiro-MeOTAD species 1 measured in chlorobenzene shows an intense band at $395 \mathrm{~nm}$ and a less intense band at ca. $300 \mathrm{~nm} .{ }^{24}$ The stepwise formation of the oxidized species can be followed by the changes of the absorption spectrum upon the addition of the oxidizing agent to the spiro-MeOTAD solution. In particular, the formation of the radical cation is evidenced by the appearance of a new absorption feature at $511 \mathrm{~nm}$ and of a weak and broad signal at ca. $700 \mathrm{~nm}$. Passing from the mono- to the dication, the intensity of the feature at ca. $510 \mathrm{~nm}$ decreases, and a quite intense absorption band at $690 \mathrm{~nm}$ is found. In addition, $\mathbf{1}^{2+}$ also absorbs in the infrared region at $1400 \mathrm{~nm} .{ }^{24}$ 


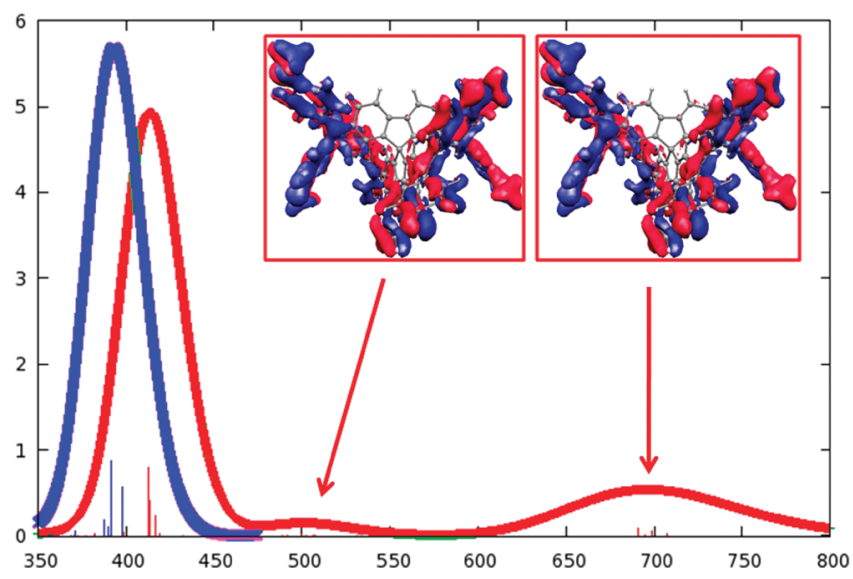

Figure 5. Comparison between the simulated absorption spectra, energies, and related oscillator strengths of the electronic transitions of $\mathbf{1}$ (blue) and $\mathbf{1}^{+}$(red). Inset: Isodensity plots of the electron density differences between the excited state and the ground state associated with the most intense transitions of the two visible absorption bands. A blue (red) color indicates a decrease (increase) of the electron density upon excitation.

The tetracation still shows the absorption feature at ca. $690 \mathrm{~nm}$, along with a new low-energy absorption band at $865 \mathrm{~nm}^{24}$

The simulated absorption spectrum of $\mathbf{1}$ is reported in Figure 4, and the calculated transitions in terms of energies, oscillator strengths, and compositions are reported in the Supporting Information. The agreement between the measured and computed absorption band maxima is excellent, in terms of both energy position and, qualitatively, intensity distribution. We computed absorption bands at 393 and $315 \mathrm{~nm}$, the latter less intense than the former, to be compared with experimental bands at 395 and $300 \mathrm{~nm}$. From the analysis of the TDDFT eigenvectors, we assign the band at $393 \mathrm{~nm}$ as originating from transitions from HOMO/ $\mathrm{HOMO}-1$ to both LUMO and LUMO +1 . These transitions have a partial charge-transfer (CT) character from the four nitrogen lone pairs of the MeOTAD ligands to the spirobifluorene core; see the corresponding charge density difference in the inset of Figure 4. The shorter-wavelength absorption band is given by transitions involving the $\mathrm{HOMO}$ as the starting state, with $\mathrm{LUMO}+7$ and LUMO +8 as final states. In particular, these transitions have $\pi-\pi^{*}$ character and originate from the fluorene phenyl moieties to the outer MeO-substituted phenyls; see the inset of Figure 4. This UV absorption band of spiro-MeOTAD falls in the same energy region as the characteristic spectroscopic feature of triphenylamine and its related substituted and derived species (ca. $300 \mathrm{~nm}) .{ }^{55,56}$

The simulated absorption spectrum of the spiro-MeOTAD monocation $\mathbf{1}^{+}$in the visible to near-IR region is reported in Figure 5, where it is compared to that of the neutral species to underline the spectral changes occurring upon oxidation. Notice that, for $\mathbf{1}^{+}$, we also calculated two low-lying transitions far in the IR region $(\sim 1750 \mathrm{~nm})$ that are not of direct interest for the present investigation and will therefore not be discussed in detail. In the computed UV-vis absorption spectrum of $\mathbf{1}^{+}$, the main absorption band is red-shifted compared to that of the neutral species (414 versus $393 \mathrm{~nm}$ ), reflecting the electronic structure picture discussed above (Figure 2), still maintaining the same partial CT character. Compared to the absorption spectrum of the neutral species, that of $\mathbf{1}^{+}$exhibits two new bands at 696 and

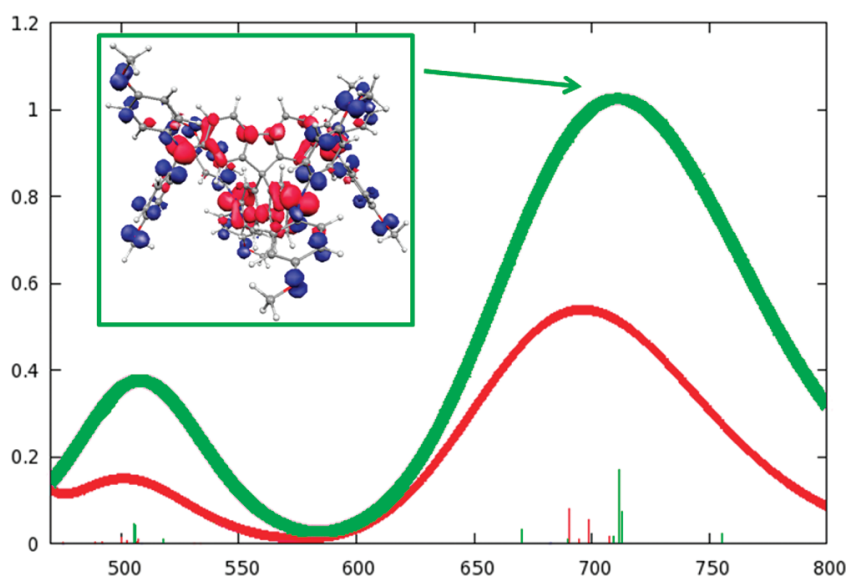

Figure 6. Comparison between the simulated absorption spectra, energies, and related oscillator strengths of the electronic transitions of $\mathbf{1}^{+}$(red) and $\mathbf{1}^{2+}$ (green). Inset: Isodensity plot of the electron density difference between the excited state and the ground state associated with the most intense transition of the NIR absorption band. A blue (red) color indicates a decrease (increase) of the electron density upon excitation.

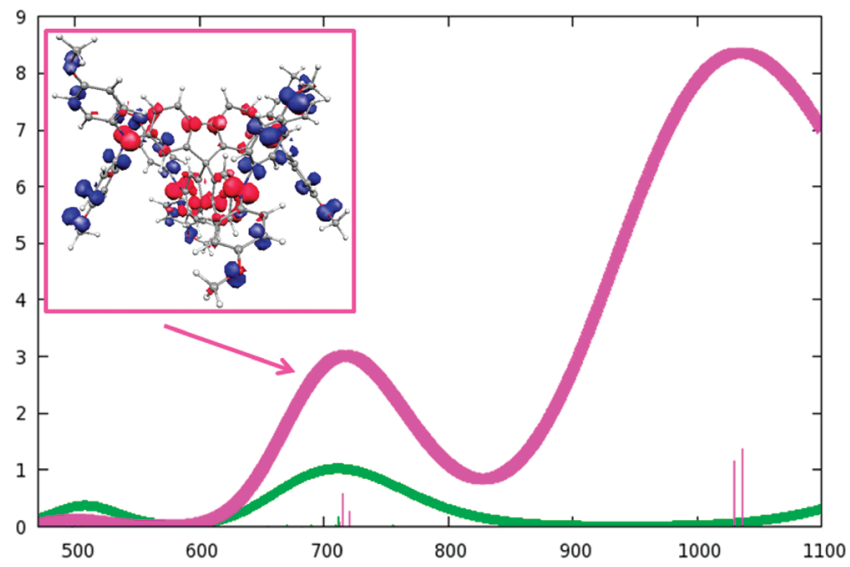

Figure 7. Comparison between the simulated absorption spectra, energies, and related oscillator strengths of the electronic transitions of $\mathbf{1}^{2+}$ (green) and $\mathbf{1}^{4+}$ (magenta). Inset: Isodensity plot of the electron density difference between the excited state and the ground state associated with the most intense transition of the NIR absorption band. A blue (red) color indicates a decrease (increase) of the electron density upon excitation.

$501 \mathrm{~nm}$, which represent the experimental signature of the radical cation. The agreement between the experimental and computed spectra of $\mathbf{1}^{+}$is excellent, even though the relative intensity of the simulated features at $501 \mathrm{~nm}$ and $696 \mathrm{~nm}$ is reversed with respect to the experimental spectrum. Analysis of the TDDFT eigenvectors reveals that the longer-wavelength absorption band $(696 \mathrm{~nm})$ takes place within the $\beta$-manifold orbitals, involving the LUMO as the arriving state. The transitions composing this band essentially involve charge redistribution from the $\mathrm{MeO}-$ substituted phenyl groups to the fluorene phenyls, along with $\pi-\pi^{*}$ transitions within the fluorene moiety (see the inset of Figure 5). The band at $501 \mathrm{~nm}$ originates from two transitions, one having as starting states the low-lying HOMOs (306 $\beta$ and $312 \beta$ ) localized on the $\mathrm{MeO}$-substituted phenyls and the LUMO of $\pi^{*}$ character within the MeO-substituted ligands as the 
arriving state and the other one involving HOMO $-1 / \mathrm{HOMO}$ as starting states and LUMO and LUMO +1 as arriving states, again within the active $\beta$-orbital manifold.

The isodensity plots of the electron density differences between the excited states and the ground state associated with the two lowest-energy absorption bands show that the involved transitions are entirely confined within the $\mathrm{MeO}$-substituted aromatic framework (see Figure 5). In analogy with the related triphenylamine (TPA) species, the spectroscopic signature of the formation of the $\mathrm{TPA}^{\bullet+}$ radical cation is the appearance of two lowenergy absorption bands at ca. 550 and $660 \mathrm{~nm} .{ }^{57}$ Because of the structural and electronic complexity of spiro-MeOTAD with respect to a single TPA, the absorption maximum wavelengths of its lower-energy absorption bands are slightly different from those of TPA. Moreover, it has been reported that substituents in the para positions of phenyls and highly conjugated substituents, such as the spiro core in our case, sizably affect the visible absorption bands of TPA. ${ }^{55}$

The absorption spectrum of spiro-MeOTAD dication $\mathbf{1}^{2+}$ was simulated and is reported in Figure 6; for comparison, the corresponding monocation absorption spectrum has also been reported. We computed the two low-energy absorption bands at 711 and $507 \mathrm{~nm}$, and the intensity of the two absorption bands increased with respect to that of the corresponding bands of $\mathbf{1}^{+}$(see Figure 6). From an analysis of the TDDFT eigenvectors, we found that the two absorption bands can be assigned similarly to those of $\mathbf{1}^{+}$: The band computed at $711 \mathrm{~nm}$ has $\pi-\pi^{*}$ character and is characterized by transitions from the outer part of the system to the spiro phenyls, whereas the band at $511 \mathrm{~nm}$ together with the $\pi-\pi^{*}$ shows a partial $n-\pi^{*}$ character and occurs within the MeO-substituted aromatic framework.

Finally, we simulated the absorption spectrum of the product of the four-electron oxidation process, $\mathbf{1}^{4+}$, which is compared to that of $\mathbf{1}^{2+}$ in Figure 7. $\mathbf{1}^{4+}$ exhibits a band in the near-infrared region at $1035 \mathrm{~nm}$. Moreover the two bands present in the other investigated species were computed for $1^{4+}$ at 717 and $500 \mathrm{~nm}$. It is worth noting that the intensity of the absorption band at ca. $700 \mathrm{~nm}$ continued to increase compared to the same spectral feature of the cation and dication species, whereas the absorption band at $500 \mathrm{~nm}$ tended to disappear (see Figure 7), consistent with what was observed experimentally. The intense band in the NIR range originates from transitions involving $\mathrm{HOMO}$ and $\mathrm{HOMO}-1$ as starting states and LUMO and LUMO +1 as final states (see Figure 2). We therefore assigned this band as a mixed $n / \pi-\pi^{*}$ one. The band at $717 \mathrm{~nm}$ was assigned as a $\pi-\pi^{*}$ one, analogously to the corresponding bands of $\mathbf{1}^{+}$and $\mathbf{1}^{2+}$, originating from the outer part of the system to the central spiro phenyls. On the other hand, the less intense spectral feature at $500 \mathrm{~nm}$ shows the $n-\pi^{*}$ component already computed in the absorption spectra of the mono- and dications.

\section{SUMMARY AND CONCLUSIONS}

We have investigated the structural, electronic, and optical properties of $2,2^{\prime} 7,7^{\prime}$-tetrakis-( $N, N$-di- $p$-methoxyphenyl amine)$9,9^{\prime}$-spirobifluorene, which is the most efficient hole-transporting material employed in solid-state dye-sensitized solar cells. Whereas the neutral spiro-MeOTAD absorbs light in the UV region of the spectrum, its oxidized forms exhibit rather strong absorptions throughout the visible and near-IR ranges. Because absorption of the oxidized species takes place in the same spectral range where sensitizers absorb light, an unwanted filtering effect results in reduced photocurrent. In an effort to design new and more transparent HTMs in the oxidized state, a deeper understanding of the optical properties of neutral spiro-MeOTAD and its oxidized forms, identifying the underlying electronic transitions, is essential.

We have thus simulated the absorption spectra of neutral and oxidized spiro-MeOTAD species and characterized the corresponding one-electron oxidation steps. For the isolated molecule, we found two well-resolved oxidation processes, separated by ca. $0.2 \mathrm{eV}$, nicely matching with the available experimental observations. For the neutral and oxidized spiro-MeOTAD species, we were able to reproduce the main spectral features to a high degree of accuracy and assign the corresponding electronic transitions. In particular, for the oxidized species, we were able to reproduce the appearance in the optical absorption spectrum of new features at $\sim 500$ and $700 \mathrm{~nm}$, which are also characteristic of the TPA-like radical cations. These features, substantially overlapping with the optical absorption of typical dye sensitizers, with absorption onsets around $700 \mathrm{~nm}$, might hinder, to some extent, light absorption by the dye, thus reducing the photovoltaic performance of the related devices.

In conclusion, we expect our study to provide the required computational modeling basis for the prediction, even before their synthesis, of the optical properties of new hole-transporting materials needed to further boost the performance of solid-state dye-sensitized solar cells.

\section{ASSOCIATED CONTENT}

S Supporting Information. Molecular orbital energies. Energies, wavelengths, and compositions of the main TDDFT excitations of $1, \mathbf{1}^{+}, \mathbf{1}^{2+}$, and $\mathbf{1}^{4+}$ computed in vacuo. Absorption spectra of $\mathbf{1}$ in vacuo and ethanol solution. This material is available free of charge via the Internet at http://pubs.acs.org.

\section{AUTHOR INFORMATION}

\section{Corresponding Author}

*E-mail: simona@thch.unipg.it.

\section{ACKNOWLEDGMENT}

The authors thank FP7-NMP-2009 Project 246124 "SANS" and FP7-ENERGY-2010 Project 261920 "ESCORT" for financial support. S.F. and F.D.A. thank Istituto Italiano di Tecnologia, Project Seed 2009 "HELYOS”, for a grant.

\section{REFERENCES}

(1) O’Regan, B.; Grätzel, M. Nature 1991, 353, 737-740.

(2) Grätzel, M. Nature 2001, 414, 338-344.

(3) Hagfeldt, A.; Boschloo, G.; Sun, L.; Kloo, L.; Pettersson, H. Chem. Rev. 2010, 110, 6595-6663.

(4) Grätzel, M. Inorg. Chem. 2005, 44, 6841-6851.

(5) Grätzel, M. Acc. Chem. Res. 2009, 42, 1788-1798.

(6) Listorti, A.; O'Regan, B.; Durrant, J. R. Chem. Mater. 2011, 23, 3381-3399.

(7) O’Regan, B. C.; Durrant, J. R. Acc. Chem. Res. 2009, 42, 17991808.

(8) Boschloo, G.; Hagfeldt, A. Acc. Chem. Res. 2009, 42, 1819-1826.

(9) Yanagida, S.; Yu, Y.; Manseki, K. Acc. Chem. Res. 2009, 42, $1827-$ 1838.

(10) Sapp, S. A.; Elliott, C. M.; Contado, C.; Caramori, S.; Bignozzi, C. A. J. Am. Chem. Soc. 2002, 124, 11215-11222. 
(11) Nusbaumer, H.; Moser, J.-E.; Zakeeruddin, S. M.; Nazeeruddin, M. K.; Grätzel, M. J. Phys. Chem. B 2001, 105, 10461-10464.

(12) Feldt, S. M.; Gibson, E. A.; Gabrielsson, E.; Sun, L.; Boschloo, G.; Hagfeldt, A. J. Am. Chem. Soc. 2010, 132, 16714-16724.

(13) Daeneke, T.; Kwon, T.-H.; Holmes, A. B.; Duffy, N. W.; Bach, U.; Spiccia, L. Nat. Chem. 2011, 3, 211-215.

(14) Feldt, S. M.; Cappel, U. B.; Johansson, E. M. J.; Boschloo, G.; Hagfeldt, A. J. Phys. Chem. C 2010, 114, 10551-10558.

(15) Wang, M.; Chamberland, N.; Breau, L.; Moser, J.-E.; HumphryBaker, R.; Marsan, B. t.; Zakeeruddin, S. M.; Grätzel, M. Nat. Chem. 2010, 2, 385-389.

(16) O’Regan, B.; Schwartz, D. T.; Zakeeruddin, S. M.; Grätzel, M. Adv. Mater. 2000, 12, 1263-1267.

(17) O’Regan, B.; Lenzmann, F.; Muis, R.; Wienke, J. Chem. Mater. 2002, 14, 5023-5029.

(18) Sicot, L.; Fiorini, C.; Lorin, A.; Nunzi, J. M.; Raimond, P.; Sentein, C. Synth. Met. 1999, 102, 991-992.

(19) Gebeyehu, D.; Brabec, C. J.; Sariciftci, N. S.; Vangeneugden, D.; Kiebooms, R.; Vanderzande, D.; Kienberger, F.; Schindler, H. Synth. Met. 2001, 125, 279-287.

(20) Spiekermann, S.; Smestad, G.; Kowalik, J.; Tolbert, L. M.; Grätzel, M. Synth. Met. 2001, 121, 1603-1604.

(21) Al-Dmour, H.; Taylor, D. M.; Cambridge, J. A. J. Phys. D: Appl. Phys. 2007, 40, 5034.

(22) Kudo, N.; Honda, S.; Shimazaki, Y.; Ohkita, H.; Ito, S.; Benten, H. Appl. Phys. Lett. 2007, 90, 183513-3.

(23) Jiang, K.-J.; Manseki, K.; Yu, Y.-H.; Masaki, N.; Suzuki, K.; Song, Y.-1.; Yanagida, S. Adv. Funct. Mater. 2009, 19, 2481-2485.

(24) Bach, U. Ph.D. Thesis, École polytechnique fédérale de Lausanne

(EPFL), Lausanne, Switzerland, 2000.

(25) Snaith, H. J.; Zakeeruddin, S. M.; Wang, Q.; Péchy, P.; Grätzel, M. Nano Lett. 2006, 6, 2000-2003.

(26) Kroeze, J. E.; Hirata, N.; Schmidt-Mende, L.; Orizu, C.; Ogier, S. D.; Carr, K.; Grätzel, M.; Durrant, J. R. Adv. Funct. Mater. 2006, 16, 1832-1838.

(27) Snaith, H. J.; Schmidt-Mende, L. Adv. Mater. 2007, 19, 31873200

(28) Salbeck, J.; Yu, N.; Bauer, J.; Weissörtel, F.; Bestgen, H. Synth. Met. 1997, 91, 209-215.

(29) Salbeck, J.; Weissörtel, F.; Bauer, J. Macromol. Symp. 1998, $125,121-132$.

(30) Bach, U.; Lupo, D.; Comte, P.; Moser, J. E.; Weissortel, F.; Salbeck, J.; Spreitzer, H.; Grätzel, M. Nature 1998, 395, 583-585.

(31) Snaith, H. J.; Grätzel, M. Appl. Phys. Lett. 2006, 89, 262114-3.

(32) Snaith, H. J.; Grätzel, M. Adv. Mater. 2007, 19, 3643-3647.

(33) Snaith, H. J.; Moule, A. J.; Klein, C. d.; Meerholz, K.; Friend, R. H.; Grätzel, M. Nano Lett. 2007, 7, 3372-3376.

(34) Snaith, H. J.; Petrozza, A.; Ito, S.; Miura, H.; Grätzel, M. Adv. Funct. Mater. 2009, 19, 1810-1818.

(35) Kruger, J.; Plass, R.; Cevey, L.; Piccirelli, M.; Grätzel, M.; Bach, U. Appl. Phys. Lett. 2001, 79, 2085-2087.

(36) Moule, A. J.; Snaith, H. J.; Kaiser, M.; Klesper, H.; Huang, D. M.; Grätzel, M.; Meerholz, K. J. Appl. Phys. 2009, 106, 073111-9.

(37) Huang, D. M.; Snaith, H. J.; Grätzel, M.; Meerholz, K.; Moule, A. J. J. Appl. Phys. 2009, 106, 073112-6.

(38) Snaith, H. J.; Humphry-Baker, R.; Chen, P.; Cesar, I.; Zakeeruddin, S. M.; Grätzel, M Nanotechnology 2008, 19, 424003.

(39) Nazeeruddin, M. K.; De Angelis, F.; Fantacci, S.; Selloni, A.; Viscardi, G.; Liska, P.; Ito, S.; Takeru, B.; Grätzel, M. J. Am. Chem. Soc. 2005, 127, 16835-16847.

(40) Kruger, J.; Plass, R.; Grätzel, M.; Cameron, P. J.; Peter, L. M. J. Phys. Chem. B 2003, 107, 7536-7539.

(41) Jennings, J. R.; Peter, L. M. J. Phys. Chem. C 2007, 111, 1610016104

(42) Fabregat-Santiago, F.; Bisquert, J.; Cevey, L.; Chen, P.; Wang, M.; Zakeeruddin, S. M.; Grätzel, M. J. Am. Chem. Soc. 2008, 131, 558-562.

(43) Fabregat-Santiago, F.; Bisquert, J.; Palomares, E.; Haque, S. A.; Durrant, J. R. J. Appl. Phys. 2006, 100, 034510-7.
(44) Schmidt-Mende, L.; Grätzel, M. Thin Solid Films 2006, 500, 296-301.

(45) Ding, I. K.; Tétreault, N.; Brillet, J.; Hardin, B. E.; Smith, E. H.; Rosenthal, S. J.; Sauvage, F.; Grätzel, M.; McGehee, M. D. Adv. Funct. Mater. 2009, 19, 2431-2436.

(46) Melas-Kyriazi, J.; Ding, I. K.; Marchioro, A.; Punzi, A.; Hardin, B. E.; Burkhard, G. F.; Tétreault, N.; Grätzel, M.; Moser, J.-E.; McGehee, M. D. Adv. Energy Mater. 2011, 1, 407-414.

(47) Poplavskyy, D.; Nelson, J. J. Appl. Phys. 2003, 93, 341-346.

(48) Yuan Chiu, K.; Xiang, Su, T.; Hong, Li, J.; Lin, T.-H.; Liou, G.-S.; Cheng, S.-H. J. Electroanal. Chem. 2005, 575, 95-101.

(49) Kirkpatrick, J.; Nelson, J. J. Chem. Phys. 2005, 123, 084703-8.

(50) Rizzo, F.; Cavazzini, M.; Righetto, S.; De Angelis, F.; Fantacci, S.; Quici, S. Eur. J. Org. Chem. 2010, 4004-4016.

(51) Frisch, M. J.; Trucks, G. W.; Schlegel, H. B.; Scuseria, G. E.; Robb, M. A.; Cheeseman, J. R.; Montgomery, J. A., Jr.; Vreven, T.; Kudin, K. N.; Burant, J. C.; Millam, J. M.; Iyengar, S. S.; Tomasi, J.; Barone, V.; Mennucci, B.; Cossi, M.; Scalmani, G.; Rega, N.; Petersson, G. A.; Nakatsuji, H.; Hada, M.; Ehara, M.; Toyota, K.; Fukuda, R.; Hasegawa, J.; Ishida, M.; Nakajima, T.; Honda, Y.; Kitao, O.; Nakai, H.; Klene, M.; Li, X.; Knox, J. E.; Hratchian, H. P.; Cross, J. B.; Bakken, V.; Adamo, C.; Jaramillo, J.; Gomperts, R.; Stratmann, R. E.; Yazyev, O.; Austin, A. J.; Cammi, R.; Pomelli, C.; Ochterski, J. W.; Ayala, P. Y.; Morokuma, K.; Voth, G. A.; Salvador, P.; Dannenberg, J. J.; Zakrzewski, V. G.; Dapprich, S.; Daniels, A. D.; Strain, M. C.; Farkas, O.; Malick, D. K.; Rabuck, A. D.; Raghavachari, K.; Foresman, J. B.; Ortiz, J. V.; Cui, Q.; Baboul, A. G.; Clifford, S.; Cioslowski, J.; Stefanov, B. B.; Liu, G.; Liashenko, A.; Piskorz, P.; Komaromi, I.; Martin, R. L.; Fox, D. J.; Keith, T.; Al-Laham, M. A.; Peng, C. Y.; Nanayakkara, A.; Challacombe, M.; Gill, P. M. W.; Johnson, B.; Chen, W.; Wong, M. W.; Gonzalez, C.; Pople, J. A. Gaussian 03; revision D.02; Gaussian Inc.: Pittsburgh, PA, 2004.

(52) Cossi, M.; Rega, N.; Scalmani, G.; Barone, V. J. Comput. Chem. 2003, 24, 669-681.

(53) García-Cañadas, J.; Fabregat-Santiago, F.; Bolink, H.J.; Palomares, E.; Garcia-Belmonte, G.; Bisquert, J. Synth. Met. 2006, 156, 944-948.

(54) Cappel, U. B.; Gibson, E. A.; Hagfeldt, A.; Boschloo, G. J. Phys. Chem. C 2009, 113, 6275-6281.

(55) Amthor, S.; Noller, B.; Lambert, C. Chem. Phys. 2005, 316, $141-152$.

(56) Bhaskar, K. R.; Bhat, S. N.; Singh, S.; Rao, C. N. R. J. Inorg. Nucl. Chem. 1966, 28, 1915-1925.

(57) Oyama, M.; Higuchi, T.; Okazaki, S. Electrochem. Solid-State Lett. 2002, 5, E1-E3. 